\title{
A Markov Model for MIMO Channel Condition Number with Application to Dual-Mode Antenna Selection
}

\author{
Ping-Heng Kuo Peter J. Smith Lee M. Garth \\ Department of Electrical and Computer Engineering, \\ University of Canterbury, \\ Christchurch, New Zealand \\ \{phk15, p.smith, 1.garth\}@elec.canterbury.ac.nz
}

\begin{abstract}
The condition number is a well known indicator of the spatial selectivity of a MIMO wireless channel. The objective of this paper is to examine the temporal behavior of this metric using a Markov model construction. Specifically, we have developed two methods to calculate analytically the transition probabilities between Markov states. These methods involve the joint density for the channel eigenvalues at two adjacent time instances and the level crossing rates (LCR) of the condition number. We then use our Markov model to investigate the behavior of dual-mode antenna selection schemes in an i.i.d. Rayleigh fading channel.
\end{abstract}

\section{INTRODUCTION}

In the context of Multiple-Input Multiple-Output (MIMO) wireless channel characterization, the spatial selectivity is often of interest. The spatial selectivity can be estimated by observing the channel condition number (or eigenvalue spread), which is defined as

$$
\kappa=\frac{\lambda_{1}}{\lambda_{m}},
$$

where $\lambda_{1}$ and $\lambda_{m}$ are the largest and smallest eigenvalues of the MIMO channel correlation matrix, respectively. Note that some authors have alternatively used the ratio of the singular values, which is just the square root of (1).

Many adaptive MIMO systems that have been proposed employ the condition number as a criterion for choosing among multiple signalling strategies. The system proposed in [1] chooses either BLAST or space-time block codes based on the instantaneous channel condition number. Extending this scheme, in [2] a "dual-mode" antenna selection scheme is outlined, which uses the condition number to choose between multiplexing and general diversity techniques. Some other systems have used the condition number to identify the current propagation environment and then to pick the best transmission method accordingly [3]. In addition to (1), sometimes the ratios of other eigenvalue pairs are also of interest. For example, in [4] researchers have used the ratio of the largest channel eigenvalue to each of the other eigenvalues in order to estimate the number of effective spatial links. All of the schemes discussed here require a feedback mechanism. To analyze the impact of feedback delay, the temporal behavior of the condition number needs to be examined.

The adaptive systems described previously quantize the condition number into multiple discrete states, and each state corresponds to a specific transmission strategy. Thus, the evolution of the condition number over time can be conveniently approximated as a finite-state Markov process. Hence, the objective of this paper is to build a Markov model for the condition number of the MIMO channel correlation matrix. Note that the i.i.d. Rayleigh fading MIMO channel is often said to be "well-conditioned", as the average condition number is much lower than for channels with a line-of-sight path or spatial correlation. In fact, however, the magnitude of the condition number can vary significantly even for an i.i.d. Rayleigh MIMO channel. We aim to develop analytical approaches for computing the transition probabilities between states for this particular baseline case.

Throughout the paper our scope is focussed on the dualmode antenna selection scheme of [2], which motivates a two-state Markov model. We propose two different methods to calculate the transition probabilities for the model: the conditional probability method and the level crossing rate (LCR) method. As required for the model construction, we also present and utilize some new results such as the joint density of the eigenvalues at two adjacent time points. We then use our Markov model to investigate the impacts of some parameters on the system behavior. In particular, the probabilities of mode selection error are of interest to us.

\section{BACKGROUND AND ASSUMPTIONS}

\section{A. Channel Model}

Consider a $\left(N_{t}, N_{r}\right)$ flat fading MIMO system with $N_{t}$ transmit and $N_{r}$ receive antennas. We assume that both the transmit and receive antenna arrays are located in richscattering environments, so the propagation channel $\boldsymbol{H}$ is an $N_{r} \times N_{t}$ matrix containing i.i.d. complex Gaussian entries with zero mean and unit variance (Rayleigh fading). Here we define $m=\min \left(N_{r}, N_{t}\right), n=\max \left(N_{r}, N_{t}\right)$ and $v=n-m$. Moreover, the joint density function for the $m$ non-negative ordered eigenvalues of the channel correlation matrix $\boldsymbol{H} \boldsymbol{H}^{\dagger}$ (or $\boldsymbol{H}^{\dagger} \boldsymbol{H}$ ) is given by [5]:

$f\left(\lambda_{1}, \ldots, \lambda_{m}\right)=\frac{\exp \left(-\sum_{i=1}^{m} \lambda_{i}\right) \prod_{i=1}^{m} \lambda_{i}^{v} \prod_{i<j}\left(\lambda_{i}-\lambda_{j}\right)^{2}}{\prod_{i=1}^{m}[(n-i) !(m-i) !]}$

In this paper this joint density is used within integral expressions to find the expectation of certain functions of the eigen- 


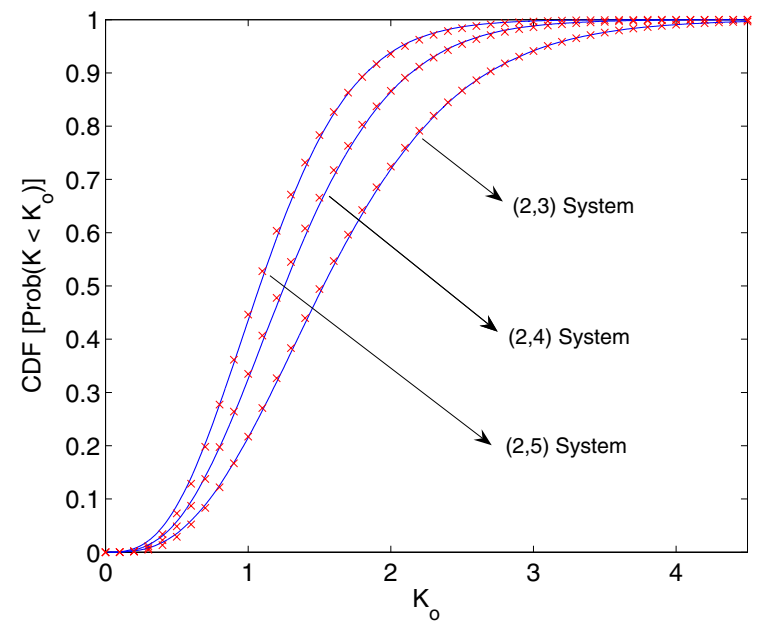

Fig. 1. Comparison of exact CDF of $K$ (line) to gamma approximation (points) for three different cases.

values. Since (2) is simply the product of powers of $\lambda_{i}$ and $\exp \left(-\lambda_{i}\right)$, closed-form integration is possible with the help of symbolic manipulation packages like MAPLE. In addition, we assume that the temporal behavior of the channel coefficients is governed by the Jakes process. Thus, the autocorrelation function for the entries of $\boldsymbol{H}$ is $\rho=J_{0}\left(2 \pi f_{D} \tau\right)$, where $J_{0}(\cdot)$ is the zeroth-order Bessel function and $f_{D}$ and $\tau$ are the Doppler frequency and time displacement, respectively.

\section{B. Gamma Approximation}

The statistical distribution of $\kappa$ is long-tailed, which often complicates any analytical approach. Therefore, we look at the logarithm of $\kappa$ (denoted $K=\log \kappa$ ) instead, which appears to be well-approximated by a gamma variable. To show this, we calculate the CDF of $K$ in closed form as follows. First, we integrate (2) with respect to $\lambda_{2}, \ldots, \lambda_{m-1}$ to obtain $f\left(\lambda_{1}, \lambda_{m}\right)$. Then, we compute the CDF of $K$ as

$$
\begin{aligned}
F_{K}\left(K_{o}\right) & =\operatorname{Prob}\left(K<K_{o}\right)=\operatorname{Prob}\left[\frac{\lambda_{1}}{\lambda_{m}}<\exp \left(K_{o}\right)\right] \\
& =\int_{0}^{\infty} \int_{\lambda_{m}}^{\lambda_{m} \exp \left(K_{o}\right)} f\left(\lambda_{1}, \lambda_{m}\right) d \lambda_{1} d \lambda_{m} .
\end{aligned}
$$

Fitting the first two moments of a gamma random variable to those of $K$, Fig. 1 demonstrates the excellent agreement between a gamma CDF and $F(K)$ for $(2,3),(2,4)$ and $(2,5)$ systems. The accuracy of the gamma approximation for some other cases has also been observed via simulations (not shown here). Along with the advantages of the gamma approximation, taking the logarithm of (1) also matches the work in [6], where the eigenvalue spread is measured on a decibel scale.

\section{Dual-Mode Antenna Selection: Two-State Markov Chain}

In this paper, we investigate the dual-mode antenna selection scheme base on a finite-state Markov model. The scheme uses the condition number as the switching criterion for choosing between multiplexing and diversity. When multiplexing is

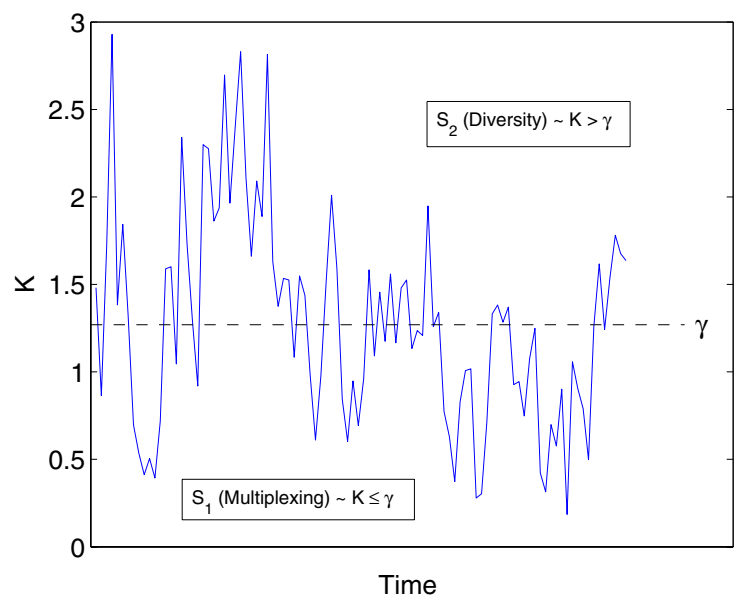

Fig. 2. Typical condition number trajectory for $(2,4)$ i.i.d. Rayleigh channel with Jakes fading. For dual-mode antenna selection scheme, condition number is quantized into two states: multiplexing $(K \leq \gamma)$ and diversity $(K>\gamma)$.

chosen, multiple independent data streams are sent simultaneously from multiple transmit antennas. For transmit diversity, on the other hand, the data stream is transmitted from the single antenna that has the best link quality. Following [2], multiplexing is chosen if $K$ is less than or equal to the threshold

$$
\gamma=\log \left[\frac{d_{\min }^{2}\left(N_{t}, D\right)}{d_{\min }^{2}(1, D)}\right],
$$

where $d_{\min }\left(N_{t}, D\right)$ and $d_{\min }(1, D)$ are the minimum Euclidean distances between points in the modulation constellations for multiplexing and diversity with rate $D$, respectively. Note that $N_{t}=1$ for the diversity scheme since only one transmit antenna is used. The system is designed assuming the total data rate $D$ is fixed, regardless of whether multiplexing or diversity is selected. Assuming that QAM is employed, $d_{\text {min }}^{2}$ can be calculated as [2]:

$$
d_{\min }^{2}\left(N_{t}, D\right)=\frac{6}{N_{t}\left(2^{D / N_{t}}-1\right)} .
$$

Hence, the condition number can be modeled by a Markov chain with two states: multiplexing $\left(S_{1}\right)$ and diversity $\left(S_{2}\right)$, as illustrated in Fig. 2. Although we concentrate on a two-state model, we must point out that our proposed analytical method is also applicable to Markov processes with more than two states.

\section{Conditional Probability Method}

In this section, we calculate the state transition probabilities using conditional probability theory. First, let $K$ and $\widehat{K}$ be $\log \kappa$ at times $t$ and $t+\tau$ respectively. The transition probability from state $S_{i}$ to state $S_{j}$ can be written as:

$$
P_{i j}=\frac{\operatorname{Prob}\left(K \in S_{i}, \widehat{K} \in S_{j}\right)}{\operatorname{Prob}\left(K \in S_{i}\right)} .
$$

Based on the discussion in the preceding section, and since the condition number is assumed to be a stationary process, the 
joint density of $K$ and $\widehat{K}$ can be approximated by a bivariate gamma distribution with common shape and scale factors, $r=$ $\mathrm{E}(K)^{2} / \operatorname{Var}(K)$ and $\theta=\mathrm{E}(K) / \operatorname{Var}(K)$. Hence, we can write the joint density $f(K, \widehat{K})$ by modifying the bivariate gamma density function in [7] as:

$$
\begin{aligned}
f(K, \widehat{K})= & \frac{(K \widehat{K})^{\frac{r-1}{2}} \theta^{r+1}}{\Gamma(r)\left(1-\rho^{\prime}\right) \rho^{\prime \frac{r-1}{2}}} \exp \left\{\frac{-(K+\widehat{K}) \theta}{1-\rho^{\prime}}\right\} \\
& \times I_{r-1}\left(\frac{2 \theta}{1-\rho^{\prime}} \sqrt{\rho^{\prime} K \widehat{K}}\right),
\end{aligned}
$$

where $\Gamma(\cdot)$ and $I_{x}(\cdot)$ represent the gamma function and $x^{t h}$ order modified Bessel function respectively, and $\rho^{\prime}$ is the correlation coefficient between $K$ and $\widehat{K}$. Alternatively, $\rho^{\prime}$ is the autocorrelation coefficient of $K(t)$ :

$$
\begin{aligned}
\rho^{\prime} & =\frac{\mathrm{E}[K(t) K(t+\tau)]-\mathrm{E}[K(t)] \mathrm{E}[K(t+\tau)]}{\sqrt{\operatorname{Var}[K(t)] \operatorname{Var}[K(t+\tau)]}} \\
& =\frac{\mathrm{E}(K \widehat{K})-\mathrm{E}(K)^{2}}{\operatorname{Var}(K)} .
\end{aligned}
$$

By using (2) to obtain $f\left(\lambda_{1}, \lambda_{m}\right), \mathrm{E}(K)$ and $\operatorname{Var}(K)$ can be calculated. Note that $\mathrm{E}(K)$ can be shown to be infinite when $v=0\left(N_{t}=N_{r}\right)$, so $\rho^{\prime}$ does not exist in this case. Therefore, here we concentrate on asymmetrical MIMO systems.

The joint moment $\mathrm{E}(K \widehat{K})$, on the other hand, must be computed using the joint density of the eigenvalues at two adjacent time points. The numerical value of $\mathrm{E}(K \widehat{K})$ can be found by integrating the product

$$
\left(\log \frac{\lambda_{1}}{\lambda_{m}}\right)\left(\log \frac{\widehat{\lambda}_{1}}{\widehat{\lambda}_{m}}\right) \times f\left(\lambda_{1}, \ldots, \lambda_{m}, \widehat{\lambda}_{1}, \ldots, \widehat{\lambda}_{m}\right)
$$

with respect to $\lambda_{1}, \ldots \lambda_{m}$ and $\widehat{\lambda}_{1}, \ldots, \widehat{\lambda}_{m}$ (2m-dimensional integration), where $\lambda_{i}$ and $\widehat{\lambda}_{i}$ are the eigenvalues at times $t$ and $t+\tau$, respectively. To the best of our knowledge, the joint density $f\left(\lambda_{1}, \ldots, \lambda_{m}, \widehat{\lambda}_{1}, \ldots, \widehat{\lambda}_{m}\right)$ is not available in the published literature. Fortunately, we have recently utilized some results for Brownian diffusion processes to derive this joint density, and it is written as [8]

$$
\begin{aligned}
f\left(\lambda_{1}, \ldots, \lambda_{m}, \widehat{\lambda}_{1}, \ldots, \widehat{\lambda}_{m}\right) \\
=\frac{\prod_{i<j}\left[\frac{1}{\alpha^{2}}\left(\widehat{\lambda}_{i}-\widehat{\lambda}_{j}\right)\right] \prod_{i<j}\left(\lambda_{i}-\lambda_{j}\right) \prod_{i=1}^{m} \lambda_{i}^{v}}{\alpha^{2 m} \prod_{i=1}^{m}[(n-i) !(m-i) !]} \\
\quad \times \exp \left(-\sum_{i=1}^{m} \lambda_{i}\right) \times G(\boldsymbol{\lambda})
\end{aligned}
$$

where

$$
G(\boldsymbol{\lambda})=\operatorname{det}\left[\begin{array}{cccc}
f\left(\widehat{\lambda}_{1} \mid \lambda_{1}\right) & f\left(\widehat{\lambda}_{2} \mid \lambda_{1}\right) & \cdots & f\left(\widehat{\lambda}_{m} \mid \lambda_{1}\right) \\
f\left(\widehat{\lambda}_{1} \mid \lambda_{2}\right) & f\left(\widehat{\lambda}_{2} \mid \lambda_{2}\right) & & \vdots \\
\vdots & & \ddots & \vdots \\
f\left(\widehat{\lambda}_{1} \mid \lambda_{m}\right) & \cdots & \cdots & f\left(\widehat{\lambda}_{m} \mid \lambda_{m}\right)
\end{array}\right]
$$

and

$$
f(\widehat{\lambda} \mid \lambda)=\frac{\alpha^{2}}{\beta^{2}}\left(\frac{\widehat{\lambda}}{\alpha^{2} \lambda}\right)^{v / 2} \exp \left[\frac{-\alpha^{2} \lambda-\widehat{\lambda}}{\beta^{2}}\right] I_{v}\left(2 \frac{\alpha}{\beta^{2}} \sqrt{\lambda \widehat{\lambda}}\right)
$$

with $\alpha=\rho, \beta=\sqrt{1-\rho^{2}}$ and $\rho=J_{0}\left(2 \pi f_{D} \tau\right)$ as before.

As all of the parameters required by the bivariate gamma density (7) are now available, each transition probability defined by (6) can be evaluated using:

$$
P_{i j}=\frac{\int_{S_{i}} \int_{S_{j}} f(K, \widehat{K}) d \widehat{K} d K}{\int_{S_{i}} f(K) d K}
$$

where $f(K)$ is approximated by the gamma density function with parameters $r$ and $\theta$.

\section{Level Crossing Rate Method}

Unfortunately, even for the simplest MIMO systems with $m=2$, the conditional probability method of Sec. III requires quadruple integration to find $\rho^{\prime}$. Hence, we propose to compute the transition probabilities using an LCR approximation in this section, which substantially reduces the number of integrations required. For the two-state model of Sec. II, the transition probabilities can be approximated by

$$
\begin{aligned}
P_{i j} & \approx \frac{\operatorname{LCR}(\gamma) \tau}{\operatorname{Prob}\left(K \in S_{i}\right)} \\
P_{i i} & =1-P_{i j}, \quad i \neq j
\end{aligned}
$$

where $i, j \in\{1,2\}$. Note that general LCR-based transition probability calculations for Markov models with more states can be found in [9] and the references therein.

Here we summarize the derivation of the LCR for $K$, but a more detailed version is available in [10]. Because the distribution of $K$ can be very accurately approximated by a gamma variable, the LCR formula for the gamma process can be applied directly [11]:

$$
\operatorname{LCR}(\gamma)=\frac{1}{2 \Gamma(r)} \sqrt{\frac{2|\ddot{R}(0)|}{\pi}}(\theta \gamma)^{r-0.5} \exp (-\theta \gamma)
$$

where $\ddot{R}(0)$ is the curvature of the autocorrelation function of the process at $\tau=0$. It has been found in [10] that when $\tau$ is sufficiently small, the difference between $\lambda_{i}$ and $\widehat{\lambda}_{i}$ can be written as

$$
\triangle \lambda_{i} \approx 2 \pi f_{D}\left[\sqrt{\lambda_{i}} Z_{i} \tau+\pi f_{D}\left(n+\Phi_{i}-\lambda_{i}\right) \tau^{2}\right]
$$

where

$$
\Phi_{i}=\sum_{k \neq i} \frac{\lambda_{i}+\lambda_{k}}{\lambda_{i}-\lambda_{k}}
$$

and $Z_{i}$ is a standard Gaussian variable, $\mathcal{N}(0,1)$, independent of the eigenvalues. By definition, $\widehat{K}=\log \left(\widehat{\lambda_{1}} / \widehat{\lambda_{m}}\right)$ and $\widehat{\lambda}=$ $\lambda+\triangle \lambda$, so (14) can be used to express the autocorrelation of $K, R(\tau)$, as a polynomial in $\tau$. Thus, the value of $\ddot{R}(0)$ can be acquired by doubling the coefficient of the $\tau^{2}$ term in $R(\tau)$. After some algebra, we have

$$
\begin{aligned}
\ddot{R}(0)= & \frac{4 \pi^{2} f_{D}^{2}}{\operatorname{Var}(K)} \mathrm{E}\left\{\log \left(\frac{\lambda_{1}}{\lambda_{m}}\right)\right. \\
& \left.\times\left[(n-1)\left(\frac{1}{\lambda_{1}}-\frac{1}{\lambda_{m}}\right)+\left(\frac{\Phi_{1}}{\lambda_{1}}-\frac{\Phi_{m}}{\lambda_{m}}\right)\right]\right\} .
\end{aligned}
$$


The LCR can be calculated by substituting (15) into (13).

As mentioned before, $\mathrm{E}(K)$ is infinite for symmetrical MIMO systems and (15) is therefore invalid in such situations, so we focus on cases with $N_{t} \neq N_{r}$. Note that the expectation in (15) can be performed in closed form over $\lambda_{2}, \ldots, \lambda_{m-1}$ due to the form of (2). Hence, only two numerical integrations are required in (15), substantially reducing the computational complexity relative to the calculation of $\mathrm{E}(K \widehat{K})$ in Sec. III. Using the LCR formula (13), the transition probabilities can be evaluated by applying (11) and (12).

\section{Simulation Results}

To verify our two methods of computing the transition probabilities, we compare our analytically calculated transition probabilities for $K$ with simulation results as a function of the threshold $\gamma$. For the simulated data, we generated $2 \times 10^{6}$ Monte Carlo samples with parameter $f_{D} \tau=0.0663$. This sample size and parameter value were used in all of the simulations in this paper unless otherwise specified. Some selected results obtained using the conditional probability method and the LCR method are plotted in Figs. 3 and 4. From these figures, we can see that although $K(t)$ itself is not a Markov process, the Markov approximation is surprisingly accurate and both methods provide good predictions of the transition probabilities.

We now use these methods to investigate the impact of feedback delay on the dual-mode antenna selection schemes. In particular, we are interested in the probability of adaptation error. The notion of using a Markov model here is similar to [9], where $P_{i j}(i \neq j)$ represents the probability that an inappropriate mode choice is made due to the channel variation during the feedback period. For simplicity, we only consider MIMO systems using two transmit antennas with data rates $D=4$ or 8 bits per signalling interval. When $D=4$, QPSK is used on both antennas for multiplexing, and 16QAM is used on the single antenna for diversity. In this case, using the formulas of Sec. II-C, we find that $\gamma=0.9163$. Similarly, when $D=8,16-\mathrm{QAM}$ is used on both antennas for multiplexing, while 256-QAM is used on the single antenna for diversity, and $\gamma=2.1413$ in this case. Note that $\gamma$ increases with $D$ [2].

We investigate the effects of adding receive antennas and increasing Doppler frequency on the transition probabilities. In Fig. 5, we plot the transition probabilities as a function of $N_{r}$. It can be observed that $P_{21}$ increases with $N_{r}$ while $P_{12}$ decreases with $N_{r}$. Thus, for systems with more receive antennas, we need to be more cautious if diversity is chosen, because it is more likely to become a multiplexing-preferred channel during the feedback delay. In Fig. 6, we see that all the transition probabilities increase with $f_{D}$, as expected.

Finally, we are interested in the overall probability of adaptation error, which can be computed as

$$
P_{e}=1-\sum_{i=1}^{2} \operatorname{Prob}\left(K \in S_{i}, \widehat{K} \in S_{i}\right) .
$$

Figure 7 reveals that $P_{e}$ for larger systems is higher when $\gamma$ is low, but the performance gets better if $\gamma$ is sufficiently high. So when $D$ (and hence $\gamma$ ) is high, systems with more receive antennas are preferred. Figure 8 shows that $P_{e}$ increases with $f_{D}$ for both values of $D$.

\section{CONCLUSIONS}

We have used a Markov chain to model the MIMO channel condition number. This is motivated by dual-mode antenna selection, which quantizes the condition number into two states: multiplexing $\left(S_{1}\right)$ and diversity $\left(S_{2}\right)$. Because $K$ can be wellapproximated by a gamma process, we have proposed two different methods for computing the transition probabilities. Both methods provide good accuracy. Furthermore, we have applied the model to study the impact of feedback delay on the adaptation errors. We have also examined the effects of increasing $N_{r}, f_{D}$ and $D$. Our simulation results show that $P_{12}$ and $P_{21}$ respond differently when a receive antenna is added. In terms of the overall adaptation error probability, the systems with more receive antennas behave better when the data rate (and hence the switching threshold) is reasonably high. In all cases, a higher Doppler frequency leads to the deterioration of the adaptation performance.

For future work, a method is needed to deal with symmetrical MIMO systems. Additionally, other propagation environments (e.g., Ricean and spatially correlated Rayleigh channels) or scenarios involving channel estimation errors should also be addressed due to their practical importance.

\section{REFERENCES}

[1] R. W. Heath and A. J. Paulraj, "Switching between multiplexing and diversity based on constellation distance," in Proc. Allerton Conf. Commu., Contr., and Comput., Monticello, IL, USA, Oct. 2000.

[2] R. W. Heath and D. J. Love, "Multimode antenna selection for spatial multiplexing systems with linear receivers," IEEE Trans. Signal Processing, vol. 53, pp. 3042-3056, Aug. 2005.

[3] A. Forenza, A. Pandharipande, H. Kim, and R. W. Heath, "Adaptive MIMO transmission scheme: exploiting the spatial selectivity of wireless channels," in Proc. IEEE VTC Spring, Stockholm, Sweden, Apr. 2005, pp. 3188-3192.

[4] S. Shim, J.-S. Choi, C. Lee, and D.-H. Youn, "Rank adaptive transmission to improve the detection performance of the BLAST in spatially correlated MIMO channel," in Proc. IEEE VTC Fall, Vancouver, BC, Canada, Sep. 24-29, 2002, pp. 195-198.

[5] I. E. Telatar, "Capacity of multi-antenna Gaussian channels," European Trans. on Telecomm. Related Technol., vol. 10, pp. 585-595, Nov. 1999.

[6] K. Sulonen, P. Suvikunnas, J. Kivinen, L. Vuokko, and P. Vainikainen, "Study of different mechanisms providing gain in MIMO systems," in Proc. IEEE VTC Fall, Orlando, FL, USA, Oct. 4-9, 2003, pp. 352-356.

[7] G. E. Oien, H. Holm, and K. J. Hole, "Impact of channel prediction on adaptive coded modulation performance in Rayleigh fading," IEEE Trans. Veh. Technol., vol. 53, pp. 758-769, May 2004.

[8] P.-H. Kuo, P. J. Smith, and L. M. Garth, "Joint density of eigenvalues of two correlated complex Wishart matrices: characterization of MIMO systems," IEEE Trans. Wireless Commun., Dec. 2006, accepted for publication.

[9] P.-H. Kuo and P. J. Smith, "On the probability of adaptation error in MIMO systems," in Proc. IEEE PIMRC, Helsinki, Finland, Sep 11-14, 2006.

[10] P.-H. Kuo, P. J. Smith, and L. M. Garth, "Level crossing analysis of MIMO eigenmodes and associated channel metrics," in preparation.

[11] R. Barakat, "Level-crossing statistics of aperture-integrated isotropic speckle," J. Opt. Soc. Am., vol. 5, pp. 1244-1247, 1988. 


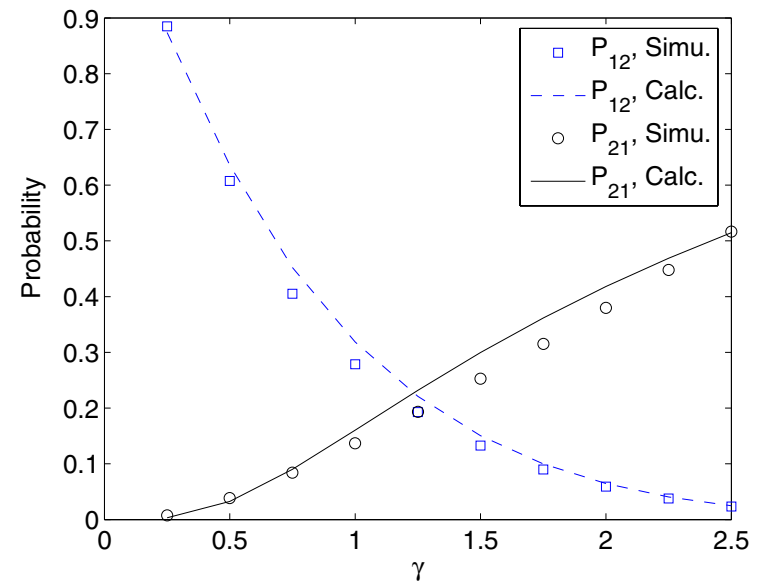

Fig. 3. Transition probabilities of $K$ for $(2,4)$ MIMO system, calculated Fig. 4. Transition probabilities of $K$ for $(3,5)$ MIMO system, calculated using the conditional probability method.

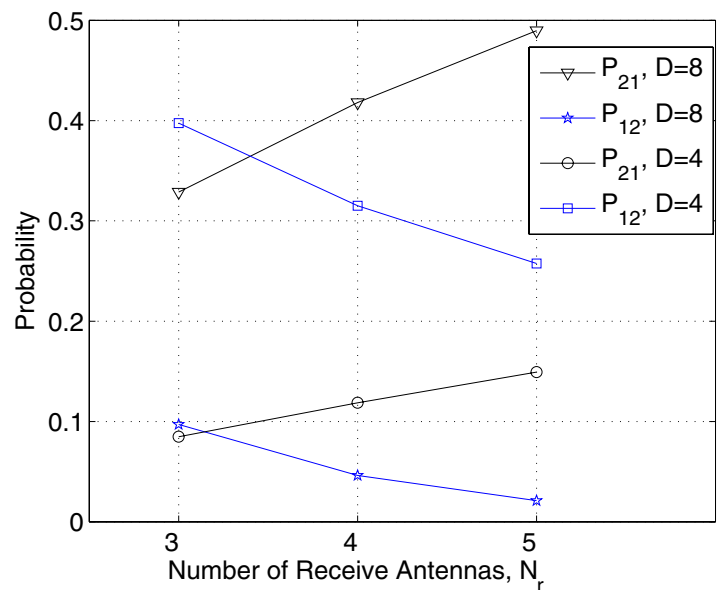

Fig. 5. Transition probabilities of $K$ for $\left(2, N_{r}\right)$ MIMO systems with different data rates.

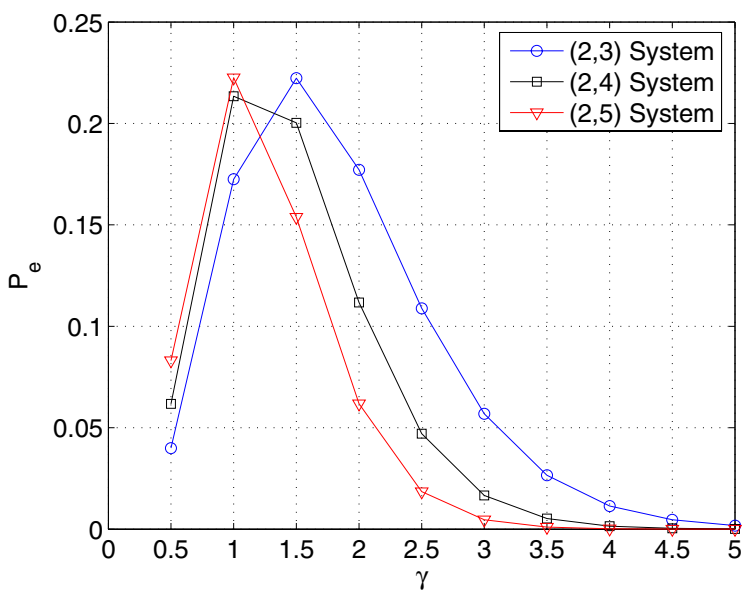

Fig. 7. Overall probability of adaptation error $P_{e}$ for $\left(2, N_{r}\right)$ MIMO systems with different switching threshold levels $\gamma$.

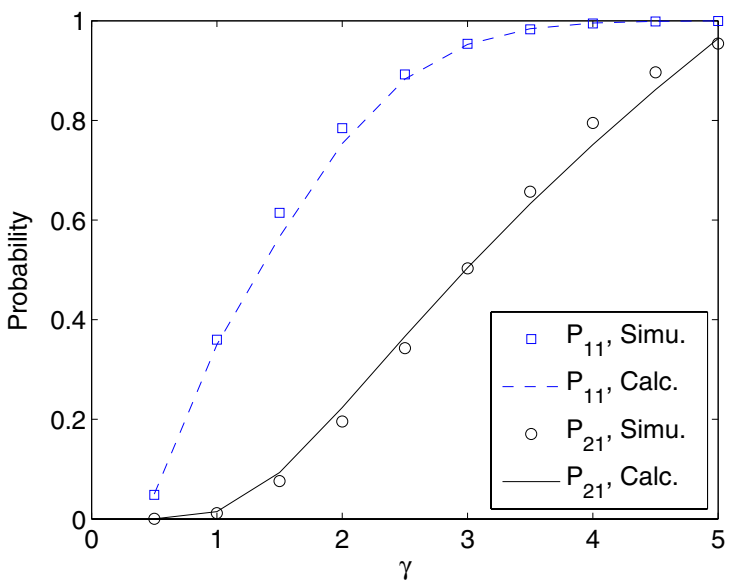

using the LCR method.

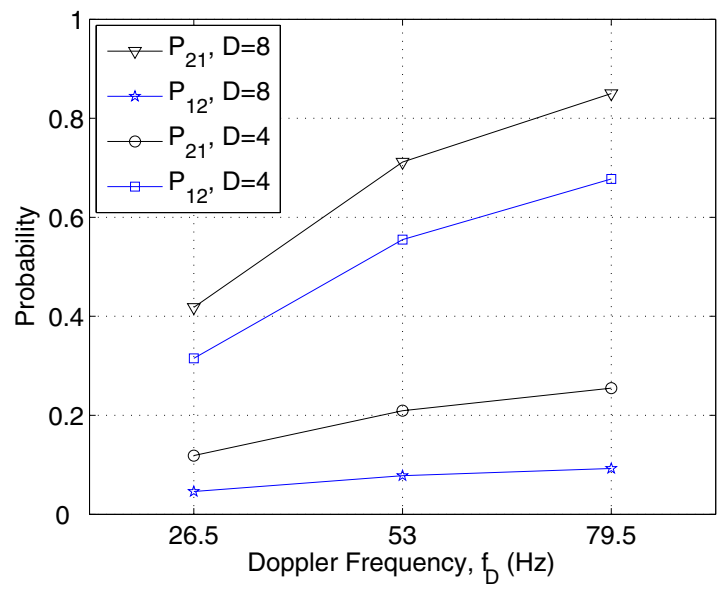

Fig. 6. Transition probabilities of $K$ for $(2,4)$ MIMO systems with different Doppler frequencies $f_{D}$ and data rates $D$.

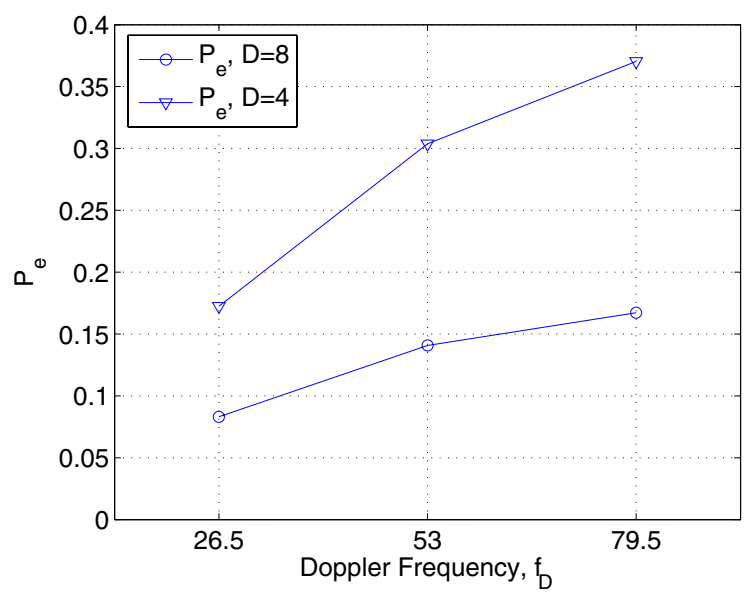

Fig. 8. Overall probability of adaptation error $P_{e}$ for $(2,4)$ MIMO systems with different different Doppler frequencies $f_{D}$ and data rates $D$. 